\title{
Placenta Disorder
}

National Cancer Institute

\section{Source}

National Cancer Institute. Placenta Disorder. NCI Thesaurus. Code C26857.

A non-neoplastic or neoplastic disorder that affects the placenta. Representative examples include chorioamnionitis, hemangioma, and choriocarcinoma. 